\title{
COMPARATIVE STUDY OF HAND GESTURE RECOGNITION SYSTEM
}

\author{
Rafiqul Zaman Khan ${ }^{1}$ and Noor Adnan Ibraheem ${ }^{2}$ \\ ${ }^{1,2}$ Department of Computer Science, A.M.U., Aligarh, India \\ ${ }^{1}$ rzk32@yahoo.co.in, ${ }^{2}$ naibraheemegmail.com
}

\begin{abstract}
Human imitation for his surrounding environment makes him interfere in every details of this great environment, hear impaired people are gesturing with each other for delivering a specific message, this method of communication also attracts human imitation attention to cast it on human-computer interaction. The faculty of vision based gesture recognition to be a natural, powerful, and friendly tool for supporting efficient interaction between human and machine. In this paper a review of recent hand gesture recognition systems is presented with description of hand gestures modelling, analysis and recognition. A comparative study included in this paper with focusing on different segmentation, features extraction and recognition tools, research advantages and drawbacks are provided as well.
\end{abstract}

\section{KEYWORDS}

Hand posture,hand gesture,human computer Interaction (HCI), Segmentation, Features Extraction, Hidden Markov Model (HMM), Artificial Neural Networks (ANNs), Fuzzy C-Mean.

\section{INTRODUCTION}

The airplane host and hostess use gestures to explain the safety guide for the passengers before the airplane takes off, since most of the passengers might unable to understand their languages especially for aged or hear-impaired passengers. Gestures used for communication between people and machines as well. Building natural interaction between human and computer required accurate hand gesture recognition system as an interface for easily human computer interaction (HCI), where the recognized gestures can be used for controlling a robot or conveying meaningful information [1].

Gestures can be static (posture) or dynamic (sequence of postures). Static gestures require less computational complexity [2] rather than dynamic gestures which are complex and for that it is suitable for real time environments [2] [3]. Acquired data in recognition system can be obtained either using spare devices such as instrumented data glove devices [4], or using the virtual hand image, both of the mentioned methods are used to extract gesture features [5]. Vision based approaches considered easy, natural and less cost comparing with glove based approaches which require the user to wear special device and be connected to the computer which hinder the naturalness of communication between the user and computes [5]. Recently a lot of reviews discussed gesture recognition systems and applications using different tools [6][7][8][9]. This work demonstrates the advancement of the gesture recognition system and it is up to date, and represents a starting point for investigators in hand gestures recognition field.

Natarajan Meghanathan, et al. (Eds): SIPM, FCST, ITCA, WSE, ACSIT, CS \& IT 06, pp. 203-213, 2012. (C) CS \& IT-CSCP 2012

DOI : $10.5121 /$ csit.2012.2320 
The paper organization is as follows: Section 2 explained the modeling of hand gesture. The analysis of hand gesture is explained in Section 3. Hand gesture recognition demonstrated in Section 4, gesture challenges discussed in Section 5. Section 6 provided a review of recent hand gesture recognition systems. Summary and conclusions presented in Section 7.

\section{Modeling Hand Gesture}

An important stage in recognition process is how to model the hand in a manner that makes postures or gestures understandable as an interface in HCI [10]. For modeling the hand, kinematic structure of the hand deemed as the base for modeling techniques [9]. There are two types of gesture modeling as classified in [10] spatial and temporal modeling. Spatial modeling consider the characteristic of posture (gesture's shape) in the environment of HCI applications [10], whereas temporal modeling relates the dynamic characteristic of the hand gesture (refers to gesture's motion) in the environments [10]. Hand Modeling in spatial domain can be implemented in 2D and 3D space [11][24].

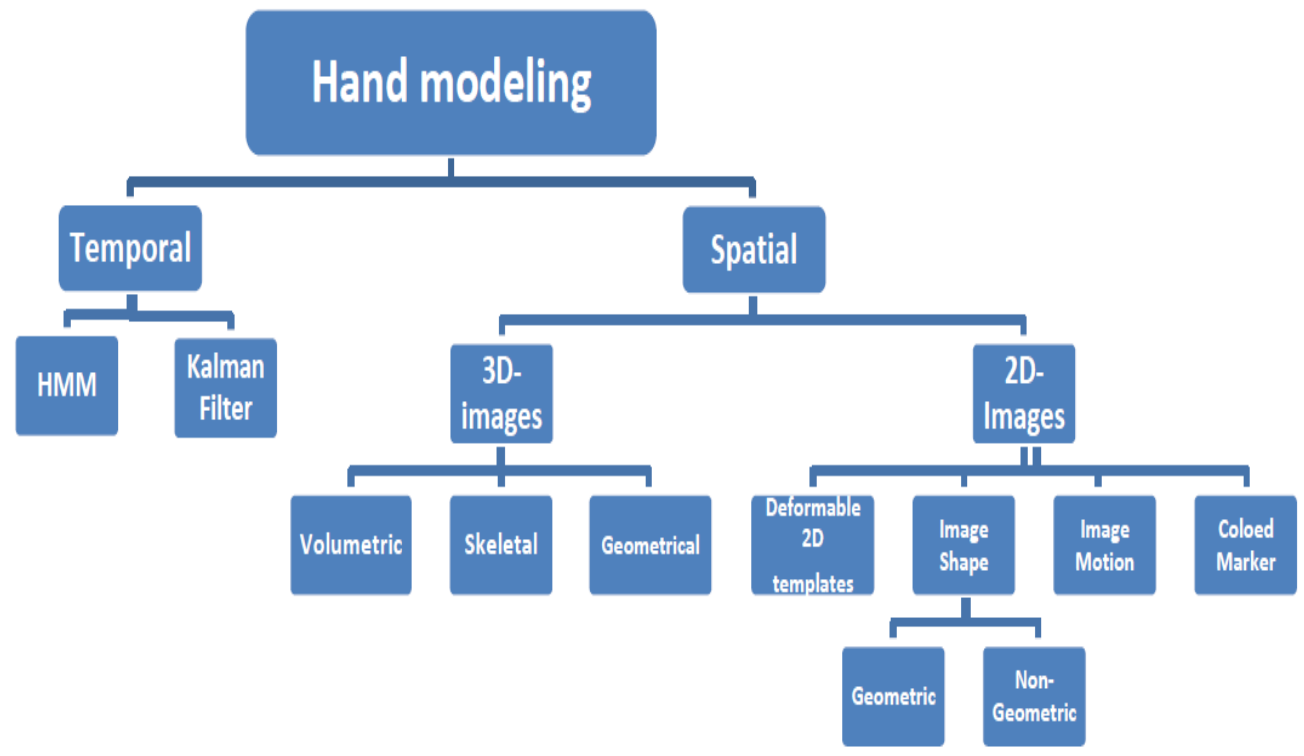

Figure 1. Hand modeling.

2D hand modeling can be represented by shape, motion, and Deformable templates [11]. Shape can be categorized into geometric models [8][24][1], and non-geometric models [1][24]. Geometric models deals with location and position features of fingertips, and palm features [24], while the non-geometric models use other shape features to models the hand such as the color [1], silhouette and textures [1], contour [9][11], edges [11], image moments [11], and eigenvectors [11], some of these features are used in features extraction and analysis as well [11]. Deformable templates or flexible models [36] provide a flexibility level of changing the shape of the object [25] to allow for little variation in the hand shape [11]. Image motion based model can be achieved with respect to color cues to track the hand, color markers are used or tracking and estimating fingers position to model the hand shape [26]. The hand shape in 3D [9] can also represented using 3D model of the hand [11][24], and can be categorized into volumetric, skeletal, and geometrical models [24][11][9]. Volumetric models are complex, computations required [9], and use a lot of parameters to represent hand shape [11], skeletal models on the other hand required less parameters to structure hand shape [11][24]. Geometrical models used widely for hand animation and real applications [26]. The geometric surfaces efficiently simulate visual 
hand image but a lot of parameters are required besides it considered as time and speed consuming process [26] [11][24]. An alternative option is to use geometric forms to approach the visual shape such as cylinders and ellipsoids [26][24][11][9]. Polygon meshes [26], and cardboard models [26] are examples of geometrical models. Figure 1 explains the techniques for hand modeling, and Figure 2 shows examples of these modeling methods.

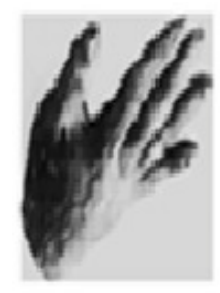

a

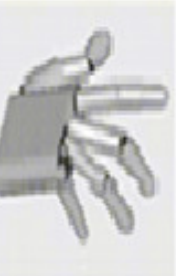

b

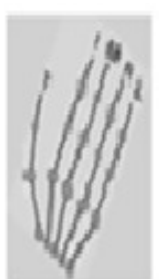

c

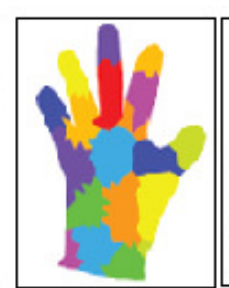

d

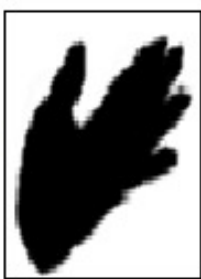

e

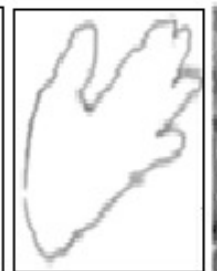

f

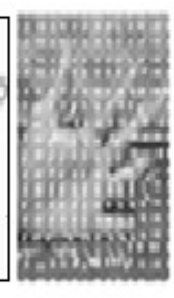

$\mathrm{g}$

Figure 2. Various hand modeling methods to represent hand posture [27]. (a) 3D volumetric model. (b) 3D geometric model. (c) 3D Skeleton model. (d) Colored marker based model. (e) Non-geometric shape model

(Binary silhouette [11]). (f) 2D deformable template model (contour [11]). (g) Motion based model.

\section{ANAlysis Hand Gesture}

After modeling the hand gesture, an analysis phase of the hand is required, which aims to estimate the necessary parameters [11] needed for gesture recognition. Two tasks are addressed for gesture analysis [11] as shown in Figure 3, firstly, features detection which relates with the extraction of useful features from the input image or input video [11], and secondly, relates with the calculation of parameters estimation model from the extracted features [11].

The detection of features required a localization of the hand to extract necessary features [9][24]. Different approaches have been applied for hand localization and features extraction [24]. Hand gesture can be localized by detecting the hand gesture from the image [11], and segmenting the hand (the foreground object) from the background which is the unwanted other objects [24]. The localization process can be categorized into two methods depending on method used for detection as described in [11], which are: color tracking and motion tracking characteristics.

Skin color provides an effective [26], and efficient [24][26] method for hand localization [26]. Segmentation based skin color are the most popular method applied for hand locating [9]. Other methods also used for same purpose, such as background subtraction [9][24], and adaptive background models [9]. The use of proper color space play a significant role in segmentation process, where some color spaces are less affected with lighting conditions such normalized color model r-g, and HS hue saturation spaces [11]. However, to provide best results of color based localization methods some limitations on the system have to be impose [9] such as the hand is the only object with skin color in the scene or the hand is the dominant object [9], besides the static background [9], and controlled lighting conditions as well [9][11]. Other researches turned to apply color glove or markers to locate hand gesture in many realistic applications [11]. Locating hand object from a sequence of image frames can be achieved using tracking motion based color property which is generally used for locating hand gesture with certain assumptions for the moving hand gesture and the background of ambient environments [11]. Since the hand is an articulated structured object, it is difficult to analyze hand motion [26]. Appearance based and 3D model based approaches can be utilized for gesture analysis to estimate parameters needed for feature extraction [26][11]. In 3D hand model two parameters are used for the analysis of kinematic shape [11] as described in [11]; angular (which is the joint angles), and linear (which is the palm dimensions). These two parameters should be evaluated properly and change when 
gestures evolve [11] in time. On the other hand appearance based models use different parameters [11], such as using optical flow for hand motion estimation [11], active contour [11], and deformable 2D template based models [11]. The fusion of color and motion cues is recently studied and could exceed some drawbacks of using these models separately [11]. Features extracted can be fingers and palm locations [24], hand position [24], and hand centroid [24]. However these features are stored in the computer and used as a template for training system data to be compared with features extracted form input image with the help of some machine learning techniques such as neural network, HMM, etc. [24].

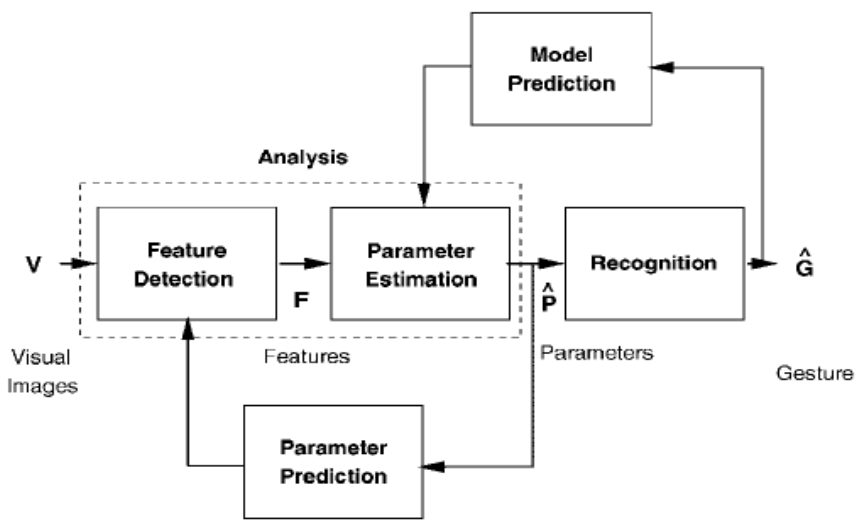

Figure 3. Analysis of hand gesture and recognition steps [11].

\section{RECOGNition HANd Gesture}

In recognition phase the input visual gesture images are recognized as a meaningful gesture depending on gesture modeling and analysis [11]. Recognition process affected with the proper selection of gesture parameters of features, and thus the accuracy of the classification [7]. For example edge detection or contour operators [7] not suitable for gesture recognition since it might lead to misclassification [7]. Neural network has been widely applied in the field of extracted the hand shape [12], and for hand gesture recognition [13][14] [15]. Hidden Markov Model (HMM) has showed excel participate as a recognition tool [16][17]. Clustering algorithms took its chance in recognition process [3], fuzzy c-mean clustering algorithm applied for controlling robot motion [2].

\section{Hand Gesture Challenges}

Hand gesture recognition system confronts many challenges as addressed in [18], these challenges are: Variation of illumination conditions; where any change in the lighting condition affects badly on the extracted hand skin region [18]. Rotation problem: this problem arises when the hand region rotated in any direction in the scene [5]. Background problem; refers to the complex background where there is other objects in the scene with the hand objects [18] and these objects might contain skin like color which would produce misclassification problem. Scale problem; this problem appears when the hand poses have different sizes in the gesture image [5]. Finally, Translation problem; the variation of hand positions in different images also leads to erroneous representation of the features [19]. 


\section{REVIEW OF GESTURE RECOGNITION SYSTEMS}

Various techniques and tools are applied for recognition hand gesture; in this section some of these methods are presented hereinafter. Li. [2] recognized hand gestures using fuzzy c-means clustering algorithm for mobile remote application. The input image is converted into HSV color space and segmented using Thresholding technique. After removing the noise from the image, 13 elements were extracted as the feature vector, first element represents the aspect ratio of the bounding box of the hand, and the rest 12 parameters represent grid cells of the image [2]. Each cell represents the mean gray level in the 3 by 4 block partition of the image, where the mean value of each cell is the average brightness of these pixels, FCM clustering algorithm used for classification gestures. The system implemented under intricate background and invariant light conditions [2]. The system trained with 120 samples, 20 samples for 6 gestures. The system implemented with recognition accuracy of $85.83 \%$ and recognition time 2-4 seconds. Hasan [18] partitioned the input image into different blocks of $23 \times 23$ pixels each, and used these blocks as a features vector. The system consists of training phase, and running phase. In training phase, several feature vectors of input gestures has been stored in a database. In testing phase the input image features vector compared with those stored in the database for classification using Euclidean distance. Since segmentation operation is imperfect, hand saturation algorithm was applied for filling the inner area of the hand gesture [18] [11]. Figure 4 shows this process.

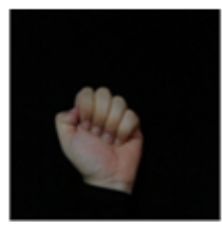

A

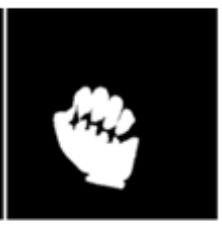

B

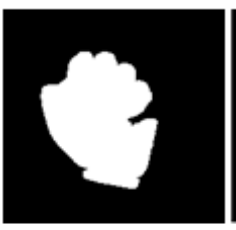

C

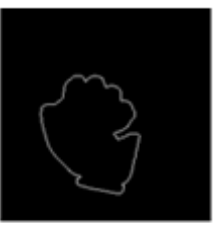

D

Figure 4. Hand Gesture Saturation process [18]. A: Original input image. B: Segmented gesture. C: Hand Gesture Saturation. D: Edge boundary.

The filling algorithm has been improved in [21] to produce Speed Border Image Initial Algorithm (SBIIA). Morphological operations for object reconstruction modified with good processing time and quality achieved as well. Dynamic structuring elements for dilation operation have been employed [21] to fix the problem unfilled objects in the previous algorithm, as seen by Figure 5.

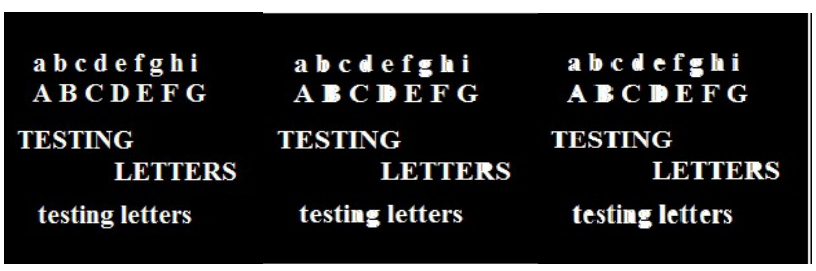

Figure 5. Modified filling algorithm (SBIIA) [21]. A: Represents the original input image, B: The processed image by the original version of the algorithm (BIIA), C: The processed image by the modified algorithm (SBIIA).

Stergiopoulou [12] suggested a new Self-Growing and Self-Organized Neural Gas (SGONG) network for hand shape approximation. For hand region detection a color segmentation technique based on skin color filter in $\mathrm{YCbCr}$ color space was used. Three features were extracted using finger identification process which determines the number of the raised fingers and characteristics of hand shape. Wysoski et al. [5] presented rotation invariant postures using boundary histogram. 
For segmentation, skin color detection filtering and clustering operation used to find the boundary for each group using ordinary contour- tracking algorithm. The boundary represented as chord's size chain which used as histograms. For classification MLP Neural Networks and DP matching were used. Kouichi [13] presented posture recognition system using neural network to recognize 42 alphabet finger symbols, and gesture recognition system to recognize 10 words. Back propagation Neural Network used for postures recognition and Elman Recurrent Neural Network for gesture recognition. The two systems were integrated in a way that after receiving the raw data, the posture system determined the sampling start time of the input image, and if it decided to be a gesture then it sent to the gesture system. Elmezain [16] proposed a system to recognize isolated and meaningful gestures for Arabic numbers (0 to 9). Gaussian Mixture Model (GMM) used for skin color detection. For features extraction, the orientation between the centroid points of current frame and previous frame were determined by vector quantization. The hand motion path recognized using different HMM topologies and BW algorithm. The system relied on zerocodeword detection model to recognize the meaningful gestures from continuous gestures (see Figure 6).

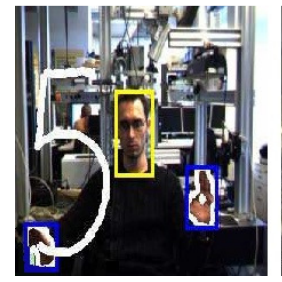

A

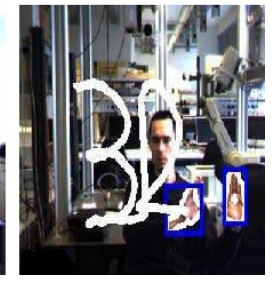

B

Figure 6. Isolation and continuous gesture representation [19]. A: path for isolation number 5. B: Continuous number 32 from connected points of hand regions.

Trigo [22] used geometric shape descriptors for gesture recognition. A webcam used to collect database image and segmented it manually. Several experiments were performed and each experiment contains one or more groups of the three features group defined; invariant moments group with seven moments; K-curvature group with the features: fingers number, angle between two fingers, and distance-radius relation; and geometric shape descriptors group with the features; aspect ratio, circularity, spreadness, roundness and solidity. Multi-layer perceptron MLP used for classification. The geometric shape descriptor group has the best performance classification. Freeman [19] presented a method for recognition gesture based on calculated local orientation histogram for each image. The system consists of training phase, and running phase. For training phase, several histograms were stored in the computer as a features vector, and in running phase, the features vector of the input gesture extracted and compared with all the feature vectors stored in computer, Euclidean distance metric used for recognized gestures.

\section{SUMMARY AND CONCLUSIONS}

Constructing an efficient hand gesture recognition system is an important aspect for easily interaction between human and machine. In this work we provided a comparative study on various gesture recognition systems with emphasis on segmentation and features detection and extraction phases which are essential for gesture modeling and analysis. Figure 7 shows the recognition rates of different recognition systems according to their appearance in the paper. Table 1 shows summaries of analysis the segmentation and features vector representation phases in hand gesture recognition systems using examples. Table 2 displays the main three steps for hand gesture recognition system; Segmentation, Features representation, and Recognition and techniques used for each phase. Finally table 3 shows the advantages and drawbacks of the some hand gesture recognition systems. 


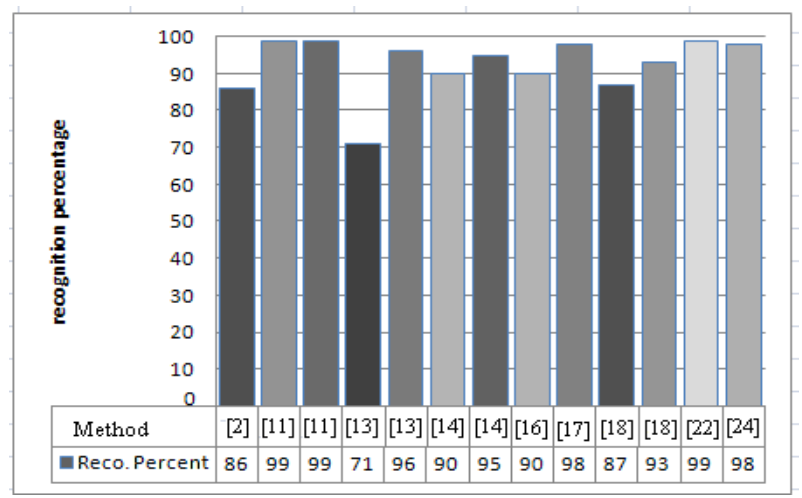

Figure 7. Recognition Rates of gesture recognition systems

Table 1. Examples of segmentation and feature vectors representation of various systems.

\begin{tabular}{|c|c|c|}
\hline Method & Segmentation & Features Vector Representation \\
\hline [2] & $\begin{array}{l}\text { A: Input image. B: Segmented } \\
\text { image. }\end{array}$ & $\begin{array}{l}\text { A: Noiseless normalized hand. B: Image } \\
\text { partitioned into } 12 \text { blocks feature vector. }\end{array}$ \\
\hline [5] & $\begin{array}{l}\text { A: Boundary representation of the } \\
\text { hand. B: Grid division. C: } \\
\text { Normalized the grid. }\end{array}$ & $\begin{array}{l}\text { Feature vector histograms represented by } \\
\text { dividing the image into number of regions } \mathrm{N} \text { in } \\
\text { a radial form. }\end{array}$ \\
\hline [12] & $\begin{array}{l}\text { A: Input image. B: Segmented } \\
\text { image. }\end{array}$ & $\begin{array}{l}\text { A: RC Angle. B: TC Angle. C: distance from } \\
\text { the palm center. }\end{array}$ \\
\hline [19] & - & The input gesture represented by a histogram. \\
\hline
\end{tabular}




\begin{tabular}{|l|l|l|}
\hline [23] & $\begin{array}{l}\text { A: Input image. B: Segmented } \\
\text { image. }\end{array}$ \\
\hline [23] & $\begin{array}{l}\text { A: Edged image. B: Features brightness } \\
\text { division. }\end{array}$ \\
\hline $\begin{array}{l}\text { A: Input image. B: Segmented } \\
\text { image. }\end{array}$ & $\begin{array}{l}\text { Features vector formed by five distances from } \\
\text { palm to all fingers and four angles between } \\
\text { those distances. }\end{array}$ \\
\hline
\end{tabular}

Table 2. Summary of segmentation, features vector representation, and recognition of hand gesture recognition systems.

\begin{tabular}{|l|l|l|l|}
\hline Method & Segmentation & Features Vector Representation & Classifier \\
\hline$[2]$ & HSV threshold & $\begin{array}{l}\text { 13 parameters as a feature vector; the } \\
\text { first parameter represents the ratio } \\
\text { aspect of the bounding hand box and } 12 \\
\text { parameters are the mean values of } \\
\text { brightness pixels in the image }\end{array}$ & $\begin{array}{l}\text { Fuzzy C-Means (FCM) } \\
\text { algorithm }\end{array}$ \\
\hline$[5]$ & thresholding & $\begin{array}{l}\text { Three features vector: Boundary } \\
\text { Chord's size FFT, Boundary Chord's } \\
\text { size, Boundary Chord's size histogram }\end{array}$ & $\begin{array}{l}\text { MLP Neural Network / } \\
\text { Dynamic Programming } \\
\text { (DP) matching }\end{array}$ \\
\hline$[12]$ & $\begin{array}{l}\text { YCbCr color } \\
\text { space }\end{array}$ & $\begin{array}{l}\text { Three angles from hand shape; RC } \\
\text { Angle, TC Angle, Distance from the } \\
\text { palm center. }\end{array}$ & Gaussian distribution \\
\hline$[13]$ & $\begin{array}{l}\text { Threshold } \\
\text { for gestures }\end{array}$ & $\begin{array}{l}\text { Back propagation } \\
\text { network / Elman recurrent } \\
\text { network }\end{array}$ \\
\hline$[16]$ & $\begin{array}{l}\text { GMM and } \\
\text { YCbCr space }\end{array}$ & $\begin{array}{l}\text { Orientation quantization } \\
\text { HMM }\end{array}$ \\
\hline$[17]$ & thresholding & $\begin{array}{l}\text { Template for posture/ hand location for } \\
\text { gesture }\end{array}$ & $\begin{array}{l}\text { structural analysis for } \\
\text { postures / HMM for } \\
\text { gestures. }\end{array}$ \\
\hline$[18]$ & $\begin{array}{l}\text { Thresholding } \\
\text { fivem }\end{array}$ & $\begin{array}{l}\text { Divide the posture image into Blocks of } \\
\text { different odd sizes ((1x1), } \\
\text { 3x3)....(23x23)) }\end{array}$ & $\begin{array}{l}\text { Euclidean Distance } \\
\text { Metric. }\end{array}$ \\
\hline$[19]$ & $\begin{array}{l}\text { thresholding } \\
\text { thresholding } \\
\text { method }\end{array}$ & $\begin{array}{l}\text { Representing hand gesture as an } \\
\text { orientation histogram }\end{array}$ & $\begin{array}{l}\text { Euclidean Distance } \\
\text { Metric. }\end{array}$ \\
\hline$[22]$ & $\begin{array}{l}\text { Segmented } \\
\text { manually } \\
\text { between these distances }\end{array}$ & $\begin{array}{l}\text { Three different features group; } \\
\text { Invariant Moments, K-curvature } \\
\text { Deometric, Shape }\end{array}$ & $\begin{array}{l}\text { Multilayer Perceptron } \\
\text { Artificial Neural } \\
\text { Network. }\end{array}$ \\
\hline$[23]$ & Quantization (LVQ). \\
\hline
\end{tabular}


Table 3. Advantages and disadvantages of hand gesture recognition systems.

\begin{tabular}{|c|c|c|}
\hline $\begin{array}{l}\text { Met } \\
\text { hod }\end{array}$ & Advantages & Disadvantages \\
\hline [2] & $\begin{array}{l}\text { (1) Speed and sufficient reliable } \\
\text { for recognition system. (2) Good } \\
\text { performance system with } \\
\text { complex background. }\end{array}$ & $\begin{array}{l}\text { (1) Irrelevant object might overlap with the } \\
\text { hand. (2) Wrong object extraction appeared if } \\
\text { the objects larger than the hand. ( } 3 \text { ) } \\
\text { Performance recognition algorithm decreases } \\
\text { when the distance greater than } 1.5 \text { meters } \\
\text { between the user and the camera is }\end{array}$ \\
\hline [5] & $\begin{array}{l}\text { (1)The radial form division and } \\
\text { boundary histogram for each } \\
\text { extracted region, overcome the } \\
\text { chain shifting problem, and } \\
\text { variant rotation problem. }\end{array}$ & $\begin{array}{l}\text { (1)The proposed method is susceptible to } \\
\text { errors, especially in shapes like square and } \\
\text { circular. }\end{array}$ \\
\hline [12] & $\begin{array}{l}\text { (1)Exact shape of the hand } \\
\text { obtained led to good feature } \\
\text { extraction. (2) Fast and powerful } \\
\text { results from the proposed } \\
\text { algorithm. }\end{array}$ & $\begin{array}{l}\text { (1) System limitations restrict the applications } \\
\text { such as; gestures are made with the right hand } \\
\text { only, the arm must be vertical, the palm is } \\
\text { facing the camera, background is plain and } \\
\text { uniform. }\end{array}$ \\
\hline [13] & $\begin{array}{l}\text { (1) Simple and active, and } \\
\text { successfully can recognize a } \\
\text { word and alphabet. (2) } \\
\text { Automatic sampling, and } \\
\text { augmented filtering data } \\
\text { improved the system } \\
\text { performance. }\end{array}$ & $\begin{array}{l}\text { (1) Required long time for Learning; several } \\
\text { hours for learning } 42 \text { characters, and four } \\
\text { days to learn ten words. (2) Large difference } \\
\text { in recognition rate for both registered and } \\
\text { unregistered people. } 98 \% \text { for registered and } \\
77 \% \text { for unregistered people. }\end{array}$ \\
\hline [16] & $\begin{array}{l}\text { (1) Recognized both isolated and } \\
\text { meaningful gestures for Arabic } \\
\text { numbers. }\end{array}$ & (1) Recognition limited to numbers only. \\
\hline [17] & $\begin{array}{l}\text { (1) The system successfully } \\
\text { recognized static and dynamic } \\
\text { gestures. (2) Could be applied } \\
\text { on a mobile robot control. }\end{array}$ & $\begin{array}{l}\text { (1) The system doesn't reflect the dynamic } \\
\text { gesture characteristics. }\end{array}$ \\
\hline [18] & $\begin{array}{l}\text { (1) Hand Saturation algorithm } \\
\text { kept the hand object as a } \\
\text { complete area. }\end{array}$ & $\begin{array}{l}\text { (1) The edge method less effective since some } \\
\text { important features was lost. }\end{array}$ \\
\hline [19] & $\begin{array}{l}\text { (1) Simple, fast, and easy to } \\
\text { implement. (2) Can be applied on } \\
\text { real system and play games. }\end{array}$ & $\begin{array}{l}\text { (1)Similar gestures have different orientation } \\
\text { histograms. (2) Different gestures have similar } \\
\text { orientation histograms. (3) Any objects } \\
\text { dominate the image will be represented as } \\
\text { orientation histograms. }\end{array}$ \\
\hline$[22]$ & $\begin{array}{l}\text { (1) Three different groups of } \\
\text { features are examined to decide } \\
\text { the good performance group. }\end{array}$ & $\begin{array}{l}\text { (1) The database samples, variation in scale, } \\
\text { translation and rotation led to a } \\
\text { misclassification in the. }\end{array}$ \\
\hline$[23]$ & (1) Used wool glove not costly & $\begin{array}{l}\text { (1) The feature vector with highest score } \\
\text { evaluated first by the classifier then the system } \\
\text { selects that features hypothesis. }\end{array}$ \\
\hline
\end{tabular}




\section{REFERENCES}

[1] G. R. S. Murthy \& R. S. Jadon. (2009) "A Review of Vision Based Hand Gestures Recognition," International Journal of Information Technology and Knowledge Management, Vol. 2, No. 2, pp 405410 .

[2] Xingyan Li. (2003) "Gesture Recognition Based on Fuzzy C-Means Clustering Algorithm", Department of Computer Science. The University of Tennessee Knoxville.

[3] S. Mitra, \& T. Acharya. (2007) "Gesture Recognition: A Survey” IEEE Transactions on systems, Man and Cybernetics, Part C: Applications and reviews, Vol. 37, No. 3, pp 311- 324, doi: 10.1109/TSMCC.2007.893280.

[4] Laura Dipietro, Angelo M. Sabatini \& Paolo Dario (2008) "Survey of Glove-Based Systems and their applications," IEEE Transactions on systems, Man and Cybernetics, Vol. 38, No. 4, pp 461-482.

[5] Simei G. Wysoski, Marcus V. Lamar, Susumu Kuroyanagi, \& Akira Iwata (2002) "A Rotation Invariant Approach On Static-Gesture Recognition Using Boundary Histograms And Neural Networks," IEEE 9th International Conference on Neural Information Processing, Singapura.

[6] Rafiqul Zaman Khan1 \& Noor Adnan Ibraheem, (2012) "Survey on Gesture Recognition for Hand Image Postures", International Journal of Computer and Information Science, Vol. 5, No. 3, pp 110121. doi: $10.5539 /$ cis.v5n3p110

[7] Joseph J. LaViola Jr. (1999) “A Survey of Hand Posture and Gesture Recognition Techniques and Technology", Master Thesis, NSF Science and Technology Center for Computer Graphics and Scientific Visualization, USA.

[8] Thomas B. Moeslund \& Erik Granum (2001) "A Survey of Computer Vision-Based Human Motion Capture,” Elsevier, Computer Vision and Image Understanding, Vol. 81, pp 231-268.

[9] Ali Erol, George Bebis, Mircea Nicolescu, Richard D. Boyle \& Xander Twombly, (2007) "Visionbased hand pose estimation: A review", Elsevier Computer Vision and Image Understanding, Vol. 108 , pp 52-73.

[10] P. Garg, N. Aggarwal \& S. Sofat, (2009). "Vision Based Hand Gesture Recognition," World Academy of Science, Engineering and Technology, Vol. 49, pp 972-977.

[11] Pavlovic, V. I., Sharma, R. \& Huang, T. S. (1997) "Visual Interpretation of Hand Gestures for Human- Computer Interaction: A Review". IEEE Transactions on Pattern Analysis And Machine Intelligence, Vol. 19, No. 7, pp 677- 695, doi; 10.1109/34.598226

[12] E. Stergiopoulou \& N. Papamarkos. (2009) "Hand gesture recognition using a neural network shape fitting technique," Elsevier Engineering Applications of Artificial Intelligence, Vol. 22, No. 8, pp 1141-1158, doi: 10.1016/j.engappai.2009.03.008

[13] Kouichi M. \& Hitomi T. (1999) “Gesture Recognition using Recurrent Neural Networks, ” ACM SIGCHI conference on Human factors in computing systems: Reaching through technology (CHI '91), pp 237-242, doi: 10.1145/108844.108900

[14] Manar Maraqa \& Raed Abu-Zaiter, (2008) "Recognition of Arabic Sign Language (ArSL) Using Recurrent Neural Networks," IEEE First International Conference on the Applications of Digital Information and Web Technologies, (ICADIWT 2008), pp 478-48. Doi: 10.1109/ICADIWT.2008.4664396

[15] Tin Hninn H. Maung. (2009) "Real-Time Hand Tracking and Gesture Recognition System Using Neural Networks," World Academy of Science, Engineering and Technology, Vol. 50, pp 466- 470.

[16] Mahmoud E., Ayoub A., J"org A., \& Bernd M., (2008) "Hidden Markov Model-Based Isolated and Meaningful Hand Gesture Recognition", World Academy of Science, Engineering and Technology, Vol. 41, pp 393-400.

[17] Min B., Yoon, H., Soh, J., Yangc, Y., \& Ejima, T. (1997) "Hand Gesture Recogrution Using Hidden Markov Models". IEEE International Conference on computional cybernetics and simulation, Vol. 5, pp 4232-4235. Doi: 10.1109/ICSMC.1997.637364 
[18] M. M. Hasan \& P. K. Mishra, (2011) “Performance Evaluation of Modified Segmentation on Multi Block For Gesture Recognition System”, International Journal of Signal Processing, Image Processing and Pattern Recognition, Vol. 4, No. 4, pp 17-28.

[19] W. T. Freeman \& Michal R., (1995) “Orientation Histograms for Hand Gesture Recognition”, IEEE International Workshop on Automatic Face and Gesture Recognition, Zurich, pp.

[20] M. M. Hasan , \& P. K. Mishra, (2012) “Improving Morphology Operation for 2D Hole Filling Algorithm”, International Journal of Image Processing (IJIP), Vol. 6, No. 1, pp. 635-646.

[21] T. R. Trigo \& S. R. M. Pellegrino, (2010) “An Analysis of Features for Hand-Gesture Classification”, 17th International Conference on Systems, Signals and Image Processing (IWSSIP 2010), pp 412415.

[22] M. M. Hasan \& P. K. Mishra, (2011) "HSV Brightness Factor Matching for Gesture Recognition System”, International Journal of Image Processing (IJIP), Vol. 4, No. 5, pp 456-467.

[23] Luigi Lamberti \& Francesco Camastra, (2011) "Real-Time Hand Gesture Recognition Using a Color Glove", Springer 16th international conference on Image analysis and processing: Part I (ICIAP'11), pp 365-373.

[24] Mokhtar M. Hasan, and Pramod K. Mishra, (2012) "Hand Gesture Modeling and Recognition using Geometric Features: A Review", Canadian Journal on Image Processing and Computer Vision Vol. 3, No.1.

[25] T. F. Cootes, C. J. Taylor, D. H. Cooper, And J. Graha, (1995) “Active Shape Models-Their Training and Application", Computer Vision And Image Understanding, Vol. 61, No. 1, pp 38-59.

[26] Ying Wu and Thomas S. Huang, (2001) "Hand Modeling, analysis and Recognition”, IEEE Signal Processing Magazine, Vol. 18, No. 3, pp 51-60. doi: http://dx.doi.org/10.1109/79.924889

[27] Mohamed B. Kaaniche, (2009) “Human Gesture Recognition” PowerPoint slides.

\section{Authors}

Dr. Rafiqul Zama Khan obtained his B.Sc degree from M.J.P Rohilkhand University, Bareilly, M.Sc and M.C.A from Aligarh Muslim University Aligarh, and his Ph.D. from Jamia Hamdard University, New Delhi. He has 18 years of rich teaching experience of various reputed National (Pune University, Jamia Hamdard University) \& International Universities (King Fhad University of Petroleum \& Minerals, Dharan, K.S.A; Ittihad University, U.A.E). Presently he is working as an Associate Professor in Department of Computer Science, Aligarh Muslim University Aligarh (U.P), India. He worked as a Head of the Department of Computer Science at Poona College, University of Pune. He also worked as a

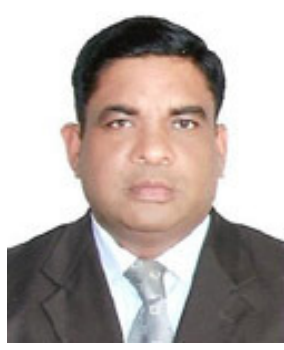
Chairman of the Department of Computer Science, at Aligarh Muslim University, Aligarh, India. He is also working as a $\mathrm{PhD}$ guide of several students. He has published more than 25 research papers in International/National Journals. He is the member of Editorial Board of number of International Journals.

Noor Adnan Ibraheem: Received her B.Sc. and M.Sc. in computer science from BGU in 2001 and 2005 respectively, she is currently a Ph.D. student at Aligarh Muslim University, Aligarh, Uttar Pradesh, India. Her research interests include computer vision, image processing, and artificial intelligent.

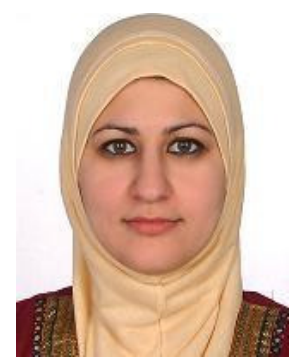

